\title{
The unification of gravity and quantum physics due to the isospin of Dirac particles
}

\author{
Engel Roza \\ Philips Research Labs, Eindhoven, The Netherlands (retired) \\ Email: engel.roza@onsbrabantnet.nl
}

\section{Summary}

It is shown that gravity and quantum physics can be unified upon the basis of a quark description in terms of a gravitational Dirac particle. It requires the awareness of a second elementary dipole moment (isospin) next to the angular moment (spin) of Dirac particles and the awareness of an (unbroken) omnipresent energetic cosmological background field. The unification has been made explicit by an expression that relates the two major gravitational constants of nature (the gravitational constant and Milgrom's acceleration constant) with the two major nuclear constants of nature (the weak interaction boson and the Higgs boson).

Keywords: quantum physics; grand unification; isospin; SSB; Dirac particle; Milgrom's constant

\section{Introduction}

In his classic paper on electrons, Dirac [1] has derived expressions for two elementary dipole momenta. The first one is due to the well known elementary angular momentum, eventually dubbed as spin $S=\hbar / 2$, which manifests itself physically as a magnetic dipole moment, with a magnitude linearly proportionate to $S$. The second one is an elementary dipole moment with magnitude $\hbar / 2 c$, which is less known. It has been waived away by Dirac, because it showed up as an imaginary quantity for which he could not find a physical justification. If it would have a physical justification, one might expect that it would show up as an elementary electrical dipole moment. In present day quantum physics, quite some studies are going on, aiming to establish values for the electrical dipole moment (EDM) of electrons $[2,3,4,5]$. The main drive behind the studies is to discover a possible violation of parity symmetry and time reversal symmetry, which would give a lead for the explanation of the matter/antimatter asymmetry in the universe. Curiously, in those studies usually no reference is given to Dirac's paper, who took the pointlike format of an electron as an axiom. An electric dipole moment would show up if the center of charge in the rest frame of a particle is shifted from the center of mass. This would be rather curious for a pointlike particle. Therefore, there is a general belief that an electric dipole of an electron is nonexisting [6]. From that perspective, Dirac's reject of a physical meaning of his own discovery makes sense. However, Hestenes [7,8] in his studies on the jitter ("zitterbewegung") of electrons pointed out that the rest frame of an electron is an illusion, because an electron, like all particles, is subject to the Heisenberg uncertainty, i.e., subject to an elementary vibration. As shown in a recent article, this motion can be interpreted as an elementary linear dipole moment, next to the better known angular dipole moment [9]. This linear dipole moment gives rise to an anomalous electric dipole moment as a quantum mechanical phenomenon, which is different from an EDM (electric dipole moment) conceived as a shift between the center of mass and the center of charge. As derived by Dirac [1], it has a value 
$q \hbar / 2 m_{0} c$, where $q$ is the elementary electric charge, $\hbar$ Planck's (reduced) constant, $m_{0}$ the rest mass of the electron and $c$ the vacuum light velocity. It is much larger than the upper limit of an EDM $\left(<0.8710^{-30} q \mathrm{~m}\right)$ as quoted by the Particle Data Group (PDG). Unfortunately, for reasons as argued in [9], in atomic based experiments the anomalous electric dipole moment of an electron escapes from observation.

While possibly Dirac's second dipole moment is of limited value for electrons, it is not necessarily true for other Dirac-type particles. In particular, this might be so for a quark, conceived as a Dirac particle. Similarly as an electron, a quark has an anomalous angular dipole moment. It is usually denoted as nuclear spin. Similarly as an electron, a quark has an anomalous linear dipole moment. It can be denoted as isospin. It is quite curious that in the Standard Model of particle physics the relationship between isospin and Dirac's linear dipole moment has not been recognized or acknowledged. Instead, isospin has been defined axiomatically as an attribute on itself, just to explain phenomena that could not be explained otherwise. Formerly, to explain the difference between a neutron and a proton, presently to explain the difference between a $u$-quark and a $d$-quark.

It is my aim to show in this article that Dirac's second dipole moment has an impact on gravity as well. It might be the key to unify quantum physics with gravity. Present-day theory of quantum physics as well as present-day theory of gravity rely upon the presence of an omni-present energetic background field. In quantum physics this field is known as the "Higgs-field". It is required for explaining the origin of mass. It has an axiomatic definition, conceived in 1964,[10]. In gravity, the existence of the background field is required to explain the accelerated expansion of the universe, known since 1998, [11]. This cosmological background field has been defined on the basis of Einstein's Cosmological Constant [12]. It is also known as "dark energy". It would be odd if two different energetic background fields would exist next to each other. More logical would be if the Higgs field and the cosmological background field would be the same. To show this relation, the achievements from a recent article in this journal [13], will be combined with the results of a study on Dirac's second dipole moment [9]. The ultimate goal is to reveal a quantitatively verifiable relationship between Milgrom's acceleration constant of dark matter and the Higgs boson. To do, in paragraph 2 and 3, the cosmological background field will be profiled, followed by profiling the Higgs field in paragraphs 4 and 5.

\section{The cosmological background field}

The presence of an omnipresent background field is imposed by the vacuum solution of Einstein's Field Equation with Cosmological Constant $\Lambda$, [14]. As discussed in [15], a positive value of the Cosmological Constant in Einstein's Field Equation, under the weak limit constraint and under particular constraints for the spatial validity range, results into a modification of Poisson's equation, such that,

$$
\nabla^{2} \Phi+\lambda^{2} \Phi=-\frac{4 \pi G M}{c^{2}} \delta^{3}(r)
$$


where $\Phi$ is the gravity potential, $G$ the gravitational constant, $M$ the mass of a pointlike baryonic source, $\delta(r)$ Dirac's delta function and where $\lambda$ is related with Einstein's Cosmological Constant $\Lambda$, such that

$\lambda^{2}=2 \Lambda$

This view on the Cosmological Constant is different from the common perception that regards it as a constant of nature, while, like extensively motivated and analysed in [15], it is basically a integration constant in Einstein's Field Equation. The striking feature of (1) is the + sign associated with $\lambda^{2}$. If it were a -sign, the equation would be similar to Debije's equation for the potential of an electric pointlike charge in an ionic plasma [16]. As is well known, the solution of such equation is a shielded Coulomb field, i.e., an electric field with an exponential decay. In the gravitational equivalent (with the + sign) the near field is enhanced ("anti-screened") [13], because masses are attracting, while electric charges with the same polarity are repelling. The background field shows up as uniformly distributed Dirac/Majorana particles (with dipoles $\hbar / 2 c$ ). Within the scope of this articles, these particles will be denoted as darks for short. The particle density (hence dipole density) $P_{g 0}$ has been calculated as,

$P_{g 0}=\frac{a_{0}}{20 \pi G}$

where $a_{0}$ is Milgrom's acceleration constant. This constant has been originally been introduced defined as an empirical quantity that describes the effect of dark matter in the universe [17]. A relatively small part of the darks is clustered as baryonic particles. Another part is locally polarized by the clusters and the polarization of the remaining part remains randomly oriented. This is just the overall picture. The clusters can be locally described as gravitational systems with a central mass. Their gravitational fields are influenced by the surrounding background field. The polarization of the dark dipoles is inwardly oriented. This is different from the outward orientation that occurs in an ionized plasma. Nevertheless, the mechanism is essentially the same. It can be captured by a(n anti) decay parameter $\lambda$ for the potential of the local center of energy (the central mass). Quantitatively, the square of the anti-decay parameter appears being,

$\lambda^{2}=\frac{2 a_{0}}{5 M G}$

where $M$ is the mass of a gravitational system with a centric baryonic source, such as valid for solar systems and galaxies. Considering that the Dirac/Majorana darks are gravitational particles [9], they are characterized by an own potential field (1) with an anti-decay parameter $\lambda$ cf. (4),

$\lambda^{2}=\frac{2 a_{0}}{5 m_{D} G}$, 
where $m_{D}$ is a tiny rest mass.

\section{The potential function of a "dark"}

In the introduction paragraph I have proposed to indicate the constituents of the energetic cosmological background energy as darks. In [13], it has been argued that such a constituent is a Dirac/Majorana particle, characterized by an elementary angular dipole moment $\hbar / 2$ and an elementary linear dipole moment $\hbar / 2 c$. Not more, not less. In a way it can be conceived as a "Heisenberg molecule". Let us assign a potential field $\Phi(r)$ to this energetic particle. The near field part of this potential field is determined by the linear dipole moment attribute. Conceiving this dipole moment as the product of a unknown mass $m_{p}$ and an unknown spatial dimension $d$, the near field along the axis set up between the poles of the dipole can be expressed as,

$\Phi_{G N}(x)=\frac{G m_{p} d}{x^{2}} \rightarrow \Phi_{G N}(x)=\frac{\hbar}{2 c} \frac{\lambda^{2} G}{(\lambda x)^{2}} \rightarrow \Phi_{G N}(x)=\Phi_{0} \frac{1}{(\lambda x)^{2}} ; \quad \Phi_{0}=\frac{\hbar}{2 c} G \lambda^{2}$

Note that $\Phi_{0}$ has the dimension of energy.

The far field is the result of an effective mass from the elementary angular moment. Interpreting the angular momentum as a rotation with light speed at a fictitious radius $r_{0}=1 / g_{m} \lambda$, we have

$$
\frac{\hbar}{2}=\frac{m_{p} c}{g_{m \lambda} \lambda} \rightarrow m_{p}=g_{m} \frac{\hbar}{2} \frac{\lambda}{c}
$$

The quantity $g_{m}$ is an unknown gyrometric constant. Hence, from classical field theory,

$$
\Phi_{G F}(r)=\frac{m_{p} G}{r}=g_{m} \frac{\hbar}{2} \frac{\lambda}{c} \frac{G}{r}=g_{m} \frac{\hbar}{2} \frac{G \lambda^{2}}{c} \frac{1}{\lambda r}
$$

and, under consideration of $\Phi_{0}$ as defined in (6),

$$
\Phi_{G F}(r)=g_{m} \frac{\hbar}{2} \frac{G \lambda^{2}}{c} \frac{1}{\lambda r}=\Phi_{0} \frac{g_{m}}{(\lambda r)}
$$

Hence, the potential field of the dark along the axis set up between the dipole axis can be expressed as an energy $\Phi(\lambda x)$ such that

$$
\Phi(\lambda x)=\Phi_{0}\left\{\frac{1}{(\lambda x)^{2}}-g_{m} \frac{1}{\lambda x}\right\}
$$


Furthermore, it is known from [13] that these darks have a tiny massive energy (rest mass) to the amount of

$m_{D} c^{2}=\frac{a_{L}}{c^{2}}(\hbar c) ; \quad a_{L}=\frac{c}{t_{H}}$

where $t_{H}(\approx 13.8$ Gyear) represents the Hubble time scale. Note that the parameter freedom $\Phi_{0}$ and $\lambda$ decouple the rest mass $m_{D}$ from the fictitious mass $m_{p}$.

The volume density $N / \mathrm{m}^{3}$ is of course the same as the dipole density (3). Hence,

$N / \mathrm{m}^{3}=\frac{a_{0}}{20 \pi G}$

\section{The nuclear background field ("Higgs field")}

The Standard Model of particle physics heavily relies upon the concept of an omnipresent energetic nuclear background field, dubbed as the Higgs field. In its most simple representation, this field $\Phi$ is characterized by its Lagrangian density $U_{H}(\Phi)$. This density is heuristically defined as [10],

$U_{H}(\Phi)=\mu_{N}^{2} \frac{\Phi^{2}}{2}-\lambda_{N}^{2} \frac{\Phi^{4}}{4}$

where $\mu_{N}$ and $\lambda_{N}$ are characteristic real constants. The justification for this format is the simple fact that many predictions from the theoretical elaboration of this underlying axiom of the Standard Model are in agreement with experimental evidence. It is instructive to compare this heuristically conceived energetic background field with the background field around an electric pointlike charge in an ionized plasma [16], where the background field has the format

$U_{D B}=\lambda_{D B}^{2} \frac{\Phi^{2}}{2}$

The energetic field $\Phi$ flowing from the pointlike charge is influenced by this background field and can be derived by the overall Lagrangian density L with the generic format

$\mathrm{L}=-\frac{1}{2} \partial_{\mu} \Phi \partial^{\mu} \Phi+U(\Phi)+\rho \Phi$

where $U(\Phi)$ is the potential energy of the background field and where $\rho \Phi$ is the source term. By application of the Euler-Lagrange equation, the potential $\Phi$ of the pointlike source of this (Debije) field can be derived as, 
$\Phi_{\mathrm{DB}}=\Phi_{0} \frac{\exp \left(-\lambda_{D B} r\right)}{\lambda_{D B} r}$

with $\Phi_{0}=Q \lambda / 4 \pi \varepsilon_{0}$, where $Q$ is the electric charge and $\varepsilon_{0}$ the vacuum electric permittivity.

Supposing that a quark is an energetic pointlike source, we may try to establish its potential, thereby expecting it being influenced by the energetic background field. Unfortunately the particular format of this (broken) field prevents deriving an analytical solution $\Phi(r)$ of from (15) subject to (13). However, a numerical procedure allows deriving a two-parameter expression for $\Phi(r)$ that closely approximates a true analytical solution. The result is [18],

$\Phi(r)=\Phi_{0} \frac{\exp [-\lambda r]}{\lambda r}\left(\frac{\exp [-\lambda r]}{\lambda r}-1\right)$ with $\frac{1}{2} \mu_{c}^{2}=1.06 \lambda^{2}$ and $\frac{1}{4} \lambda_{c}^{2}=32.3 \frac{\lambda^{2}}{\Phi_{0}^{2}}$.

This result can be written as the sum of a far field and a near field, such that,

$\Phi(r)=\Phi_{F}(r)+\Phi_{N}(r)$ with $\Phi_{F}(r)=-\Phi_{0} \frac{\exp (-\lambda r)}{\lambda r}$ and $\Phi_{N}(r)=\Phi_{0} \frac{\exp (-2 \lambda r)}{(\lambda r)^{2}}$

Such a quark would show the characteristics as shown in Figure 1. It would imply that a quark would be repelled by any other quark under influence of the far field, but attracted by the near field, thereby giving rise to mesons as stable two-quark junctions and baryons as three-quark junctions.
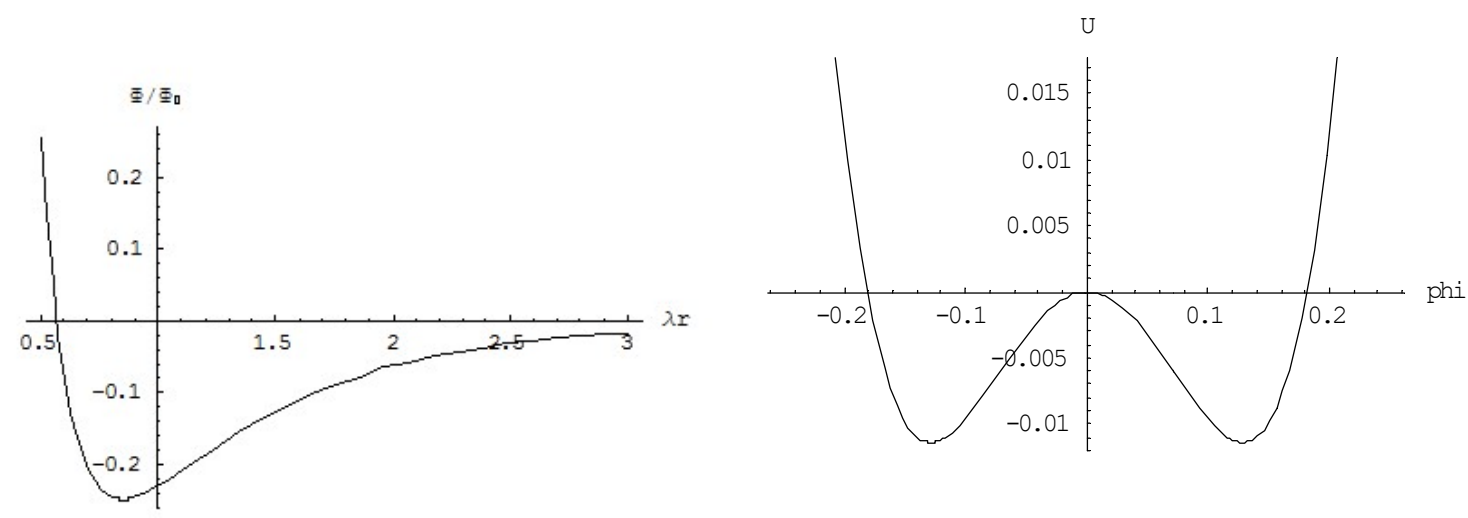

Figure 1. (Left) The quark's scalar field $\Phi / \Phi_{0}$ as a function of the normalized radius $\lambda x$; (Right) The Higgs field $U(\Phi)$ retrieved from the spatial expression.

Unfortunately, this radial symmetric solution is not viable, because the near field violates the renormalization constraint. 


\section{The potential function of a quark}

The problem can be solved by a slight modification of (17), based on the considerations as brought forward in paragraph 3. Conceiving a quark as a Dirac particle, it shows a linear dipole moment. Modeling the quark similarly as a dark, thereby adding the influence of a shielding background field, gives a potential function along the axis between the dipole poles with the format,

$\Phi(x)=\Phi_{0} \exp (-\lambda x)\left(\frac{1}{(\lambda x)^{2}}-g_{m} \frac{1}{\lambda x}\right)$

where $g_{m}$ is a dimensionless gyrometric factor. Under the condition that $g_{m} \approx 1 / 0.55$, this potential function is indistinguishable from the numerically obtained solution from the Higgs potential. Elaboration of this model has revealed that the decay parameter $\lambda$ has a direct relationship with the energy $m_{H}^{\prime}$ of the Higgs boson, such that [18].

$m_{H}^{\prime} \approx 2 \lambda(\hbar c)$.

All quarks show the same potential function. Their $\Phi_{0}$ and $\lambda$ values are related with those of the quarks in the archetype meson (pion).

\section{Three elementary Dirac-type particles}

Quarks in a meson and the cosmological darks are Dirac-type particles. In that sense they are similar to electrons. However, where the field of an electron is not affected in vacuum, the field of a quark is shielded by an energetic background field while the field of a dark is enhanced by such a field. A proper comparison between the three particle types, a generic force $F$ will be defined as the spatial derivative of a generic potential $\Phi$ in units of energy, such that for electrons, darks and quarks, respectively,

$$
\begin{aligned}
& F=q \frac{\partial}{\partial y} \Phi_{\mathrm{e}}=\frac{\partial}{\partial y} \Phi \rightarrow \Phi_{\mathrm{e}}=\frac{\Phi}{q} \\
& F=m_{0} \frac{\partial}{\partial y} \Phi_{\mathrm{G}}=\frac{\partial}{\partial y} \Phi \rightarrow \Phi_{\mathrm{G}}=\frac{\Phi}{m_{0}} \\
& F=g \frac{\partial}{\partial y} \Phi_{\mathrm{qu}}=\frac{\partial}{\partial y} \Phi \rightarrow \Phi_{\mathrm{qu}}=\frac{\Phi}{g}
\end{aligned}
$$

where $q, m_{0}$ and $g$, are the coupling factors of, respectively, an electron, a dark and a quark to, respectively, an electric potential, a gravitational potential and a nuclear potential. Electroweak unification relations the nuclear coupling factor $g$ with the electromagnetic fine structure relationship $q^{2}=4 \pi \varepsilon_{0} \hbar c g^{2}$. 


\section{Unifying gravity with quantum mechanical physics}

Because of the common origin of a dark and a quark it makes sense to relate the attributes of a quark with those of a dark. The spatial parameter $\lambda$ of a dark has been specified in (5) as,

$\lambda_{D}^{2}=\frac{2 a_{0}}{5 m_{D} G}$

where $m_{D}$ is the rest mass of a dark. Let the mass of a quark be the fictitious mass $m_{q u}$ associated with the Higgs field parameter $\lambda_{H}$. Hence from (7)

$m_{q u}=g \frac{\hbar}{2 c} \lambda_{q u}$.

The common origin of the quark and the dark allows identifying the Higgs field as the cosmological background field and the quark's fictitious mass as the mass of a dark. Hence,

$\lambda_{q u}=\lambda_{H}=\left|\lambda_{D}\right|$ and $m_{q u}=m_{D}$.

From (22) and (23),

$\frac{a_{0}}{G}=\frac{5}{2} g \frac{\hbar}{2 c} \lambda^{3}$

This is the basic unification formula. The remaining issue is establishing an expression for $\lambda$. This is possible by considering that the spacing between the quarks in the center of frame is just the half wavelength of a boson $\hbar \omega_{W}$. The half wave length of the weak interaction boson $\hbar \omega_{W}$ is about equal to the spacing $2 d_{\min }=2 d_{\min }^{\prime} / \lambda$ between the two quarks. Hence,

$2 d_{\min }=\frac{1}{2} \alpha c T$

where $T=1 / f=\omega / 2 \pi$, and $\alpha$ a dimensionless quantity of order 1 . Considered, however, in the inertial frame, the energy of the boson is the rest mass of the meson $\hbar \omega_{\pi}$.

$2 d_{\min }=\frac{1}{2} \alpha c T=\frac{1}{2} \alpha \hbar c \frac{2 \pi}{\hbar \omega_{W}}=2 \frac{d_{\min }^{\prime}}{\lambda} \rightarrow \lambda=\frac{1}{\pi} \frac{\hbar \omega_{\pi}}{\alpha \hbar c} 2 d_{\min }^{\prime}$

The normalized spacing $d_{\min }^{\prime}$ is established by a relationship between the weak interaction boson $m_{W}^{\prime}=\hbar \omega_{W}$ and the Higgs boson $m_{H}^{\prime}=\hbar \omega_{H}$. Next to (20), we have in the meson's center of mass, 
$2 d_{\min }^{\prime}=\frac{\alpha \pi \hbar c}{\hbar \omega_{W}} \lambda$

Hence, from (20) and (28),

$d_{\min }^{\prime}=\frac{\alpha \pi m_{H}^{\prime}}{4 m_{W}^{\prime}}$

The magnitude of the normalized spacing $2 d_{\text {min }}^{\prime}$ in the archetype meson is known from the equilibrium state between the quark and the antiquark as can be determined from the quark's potential function. This equilibrium state is obtained for

$\exp \left(-d_{\min }^{\prime}\right) / d_{\min }^{\prime}=\frac{1}{2} \rightarrow d_{\min }^{\prime} \approx 0.853$

The equations (25), (27) and (29) establish the relationship between gravity and quantum physics as,

$\frac{a_{0}}{G}=\frac{5}{2} g \frac{\hbar}{2 c} \lambda^{3} ; \lambda=\frac{2}{\pi} \frac{\hbar \omega_{\pi}}{\hbar c} d_{\min }^{\prime}$.

With $\mathrm{G}=6.6710^{-11} \mathrm{~kg}^{-1} \mathrm{~m}^{3} \mathrm{~s}^{-2}, \hbar \omega_{\pi} \approx 140 \mathrm{MeV}$ (pion), , the calculated value of Milgrom's acceleration constant is $1.4410^{-10} \mathrm{~m} / \mathrm{s}^{2}$. This corresponds fairly with the present known value of Milgrom's acceleration constant $\left(1.2510^{-10} \mathrm{~m} / \mathrm{s}^{2}\right)$. In view of the high sensitivity for $\lambda$, this is rather satisfying.

Furthermore, it is of interest to note that from (29) the parameter $\alpha$ is calculated from $m_{W}^{\prime} \approx 80.4 \mathrm{GeV}$ (weak interaction boson) and $m_{H}^{\prime} \approx 127 \mathrm{GeV}$ (Higgs boson) as $\alpha \approx 0.69$. This is the same value as I have derived previously from an expression of the gravitational constant in terms of quantum mechanical parameters [18]. However, where previously the pion structure has been derived by accepting the heuristics of the Higgs potential and by assuming some unknown gluing force between the quarks in a pion, the gluing force between the quarks in the present article has been derived from the second elementary dipole moment of the quark conceived as a Dirac particle. Moreover, in [18] the broken field format of the Higgs field has been accepted as an axiom from the Standard Model. In the present article, though, it has been shown that the format of this field is the result of the interaction of the energy flow from the quark with the (unbroken) cosmological background field.

\section{Conclusion}

In this article, quarks have been described as Dirac particles subject to gravitational laws. This has been possible by recognizing that Dirac particles show a second elementary dipole moment next to the well known angular moment $\hbar / 2$, usually dubbed as spin. The theory as developed in this article is based upon the correspondence between the elementary 
constituent of the cosmological background field, dubbed as "dark", and the quark as the basic elementary nuclear particle. It has been shown that The theory, based upon this view, has resulted in the view that the quark is an elementary gravitational particle. As a result, gravity and quantum physics can be unified. The unification has been explicitly been expressed in the relationship

$$
\frac{a_{0}}{G}=\frac{5}{2} g \frac{\hbar}{2 c} \lambda^{3} ; \lambda=\frac{2}{\pi} \frac{\hbar \omega_{\pi}}{\hbar c} d_{\min }^{\prime} ; \exp \left(-d_{\min }^{\prime}\right) / d_{\min }^{\prime}=\frac{1}{2}
$$

Apart from the constants of nature, $G, g, c$ and $\hbar$, this expression contains the energetic value of the rest mass of the archetype meson (pion). The calculated value of Milgrom's acceleration constant is $1.4410^{-10} \mathrm{~m} / \mathrm{s}^{2}$. It fairly corresponds to the present known value of Milgrom's acceleration constant $\left(1.2510^{-10} \mathrm{~m} / \mathrm{s}^{2}\right)$. In view of the high sensitivity for $\lambda$, this is rather satisfying. It supports the view that it is possible to unify gravity and quantum physics on the basis of the recognition of Dirac's second dipole moment.

\section{References}

[1]] P.A.M. Dirac, Proc.Royal Soc. London, A 117, 610 (1928)

[2] https://wikipedia.org/wiki/Electron electric dipole-moment

[3] https://arxiv.org/pdf/1804.10012.pdf

[4] M. Pospelov, A. Ritz, Annals of Physics 318, 119, ArXiv: hep-ph/0504231 (2005)

[5]The ACME Collaboration, Science, 343 (6168):269 (2014); ArXiv:1310.7634 (2013)

[6] I.B. Khriplovich, S.K. Lamoreaux, CP Violation Without Strangeness Electric Dipole Moments of Particles, Atoms and Molecules, Springer Verlag (1997

[7] D. Hestenes, Found Phys. 40, doi.org/10.1007/s10701-009-9360-3 (2010)

[8] D. Hestenes, ArXiv: 0.802.2728v1 (2008)

[9] E.Roza, preprints.org, doi: 10.20944/preprints201906.0142.v2 (2019)

[10] ] D. Griffiths, Introduction to Elementary Particles, ISBN 3527406018, Wiley (2008)

]11] J.A. Frieman, M.S. Turner, D. Huterer, Dragan, Ann. Rev. Astronomy and Astrophys. 46, 385 (2008)

[12] P.J.E. Peebles, B. Ratra, Bharat (2003). Reviews of Modern Physics. 75 (2): 559, (2003)

[13] E. Roza, Astrophys. And Space Sci., 364:73, doi.org/10.1007/s10509-019-3561-9 (2019)

[14] ] www.scholarpedia.org/article/Cosmological constant

[15] E. Roza, preprints.org, doi: 10.20944/preprints201701.0076.v1 (2017)

[16] P. Debye and E. Huckel, Physik. Zetschrift, vol. 24, 9, 185 (1923)

[17] M. Milgrom, The Astrophysical Journal, 270, 365 (1983)

[18] E. Roza, Results in Physics, 6 DOI: 10.1016/j.rnp 2016.03.001 (2016) 